\title{
Cellular adhesion molecules as targets for bacterial infection
}

\author{
Christof R. Hauck*, Franziska Agerer, Petra Muenzner, Tim Schmitter \\ Zentrum für Infektionsforschung, Universität Würzburg, Röntgenring 11, D-97070 Würzburg, Germany
}

\begin{abstract}
A large number of bacterial pathogens targets cell adhesion molecules to establish an intimate contact with host cells and tissues. Members of the integrin, cadherin and immunoglobulin-related cell adhesion molecule (IgCAM) families are frequently recognized by specific bacterial surface proteins. Binding can trigger bacterial internalization following cytoskeletal rearrangements that are initiated upon receptor clustering. Moreover, signals emanating from the occupied receptors can result in cellular responses such as gene expression events that influence the phenotype of the infected cell. This review will address recent advances in our understanding of bacterial engagement of cellular adhesion molecules by discussing the binding of integrins by Staphylococcus aureus as well as the exploitation of IgCAMs by pathogenic Neisseria species.
\end{abstract}

(C) 2005 Elsevier GmbH. All rights reserved.

Keywords: Neisseria gonorrhoeae; Staphylococcus aureus; Bacterial invasion; Cell adhesion; Integrin; CEACAM

\section{Contents}

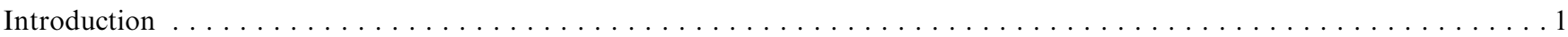

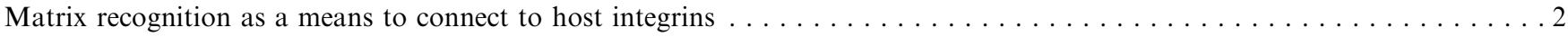

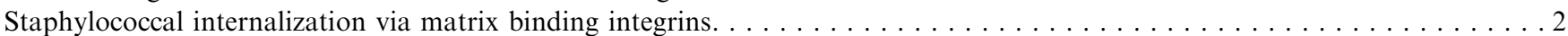

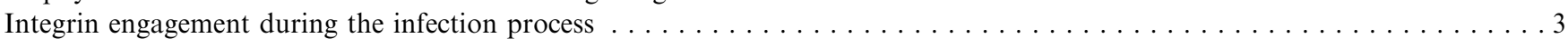

Exploitation of immunoglobulin-related cell adhesion molecules - the paradigm of pathogenic Neisseria species . . . . . . . . 4

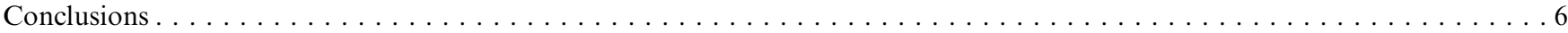

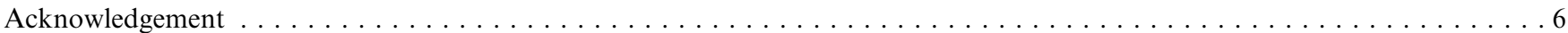

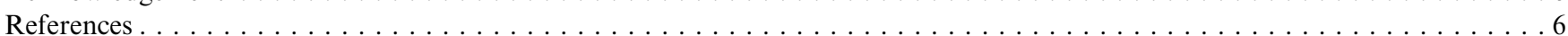

\section{Introduction}

Colonizing and establishing themselves in multicellular organisms is a challenging task for bacterial pathogens. In many cases, the microorganisms intimately associate with cells of their eukaryotic host to

\footnotetext{
*Corresponding author. Tel.: + 49931312137 ;

fax: + 49931312578 .

E-mail address: christof.hauck@mail.uni-wuerzburg.de (C.R. Hauck).
}

successfully compete with resident commensal bacteria and/or to circumvent host defense mechanisms. A dependable connection between bacterial adhesins and host surface structures is key to withstand mechanical cleansing processes ranging from eye blinking to the flushing of the urethra or the constant turnover of the mucus layer. Moreover, bacterial engagement of eukaryotic receptors often triggers a receptor-mediated internalization process that allows the microbes to access a shielded intracellular niche. Not surprisingly, this initial encounter at the cellular level is not only a 
critical determinant of pathogen-host specificity and range, but also to a large extent is a decisive point for the infection process as a whole. Strikingly, many pathogenic bacteria (and also viruses) target surfaceexposed cell adhesion molecules of the integrin, cadherin or immunoglobulin-related cell adhesion molecule (IgCAM) families. Current studies have highlighted the fact that pathogens exploit the intrinsic linkage to the actin cytoskeleton that these adhesion molecules provide, to trigger their uptake by host cells. Furthermore, it is becoming evident that receptor engagement has consequences beyond bacterial host cell association and invasion by altering gene expression in the host cell and modulating the cellular phenotype. This review will address recent advances in our understanding of bacterial engagement of cellular adhesion molecules and focuses on integrin binding by Staphylococcus aureus as well as exploitation of IgCAMs by pathogenic Neisseria species.

\section{Matrix recognition as a means to connect to host integrins}

Prominent examples of pathogenic bacteria that bind to cell adhesion molecules are listed in Table 1 . Interestingly, integrins seem to comprise prime targets for many pathogens trying to get in contact with their host cells. Whereas several pathogens express adhesins that directly engage integrins (e.g. enteropathogenic Yersinia species), other bacteria indirectly connect to integrins via the recruitment of extracellular matrix proteins. Examples include Gram-positive bacteria such as Staphylococcus aureus or Streptococcus pyogenes that have evolved a number of surface proteins binding matrix components of their host (Foster and Hook, 1998; Patti et al., 1994). These types of afimbrial adhesins include fibronectin-binding proteins $\mathrm{A}$ and $\mathrm{B}$ (FnBP-A and -B) of $S$. aureus or Sfb1 (also termed F1) of $S$. pyogenes, respectively. Both, FnBPs and Sfb1 attach the plasma component and extracellular matrix protein fibronectin to the surface of the bacteria by a tandem beta-zipper mechanism (Schwarz-Linek et al., 2003). It is thought that binding to extracellular matrix proteins allows the bacteria to colonize matrix-coated surfaces such as implanted medical devices. Furthermore, the bacterial adhesin does not only mediate direct binding to fibronectin-covered surfaces, but also utilizes fibronectin as a molecular bridge, indirectly linking the bacterial surface with the principal host fibronectin receptor, the integrin $\alpha_{5} \beta_{1}$ (Ozeri et al., 1998; Sinha et al., 1999). Upon bacterial adhesion, integrin ligation can lead to efficient internalization of the bacteria into eukaryotic cells in vitro and in vivo (Agerer et al., 2003, 2005; Brouillette et al., 2003; Ozeri et al., 1998; Sinha
Table 1. Pathogens targeting cell adhesion molecules

\begin{tabular}{|c|c|}
\hline Species & $\begin{array}{l}\text { ECM protein/ } \\
\text { receptor }\end{array}$ \\
\hline \multicolumn{2}{|l|}{ Integrins } \\
\hline Borrelia burgdorferi & $\mathrm{FN} / \beta 1$ integrins \\
\hline Mycobacterium leprae & $\begin{array}{l}\mathrm{FN}, \mathrm{LN} / \beta 1 \text { and } \beta 4 \\
\text { integrins }\end{array}$ \\
\hline Mycobacterium bovis $\mathrm{BCG}$ & $\mathrm{FN} / \beta 1$ integrins \\
\hline $\begin{array}{l}\text { Neisseria gonorrhoeae and } N \text {. } \\
\text { meningitidis }\end{array}$ & $\begin{array}{l}\mathrm{FN}, \mathrm{VN} / \beta 1 \text { and } \beta 3 \\
\text { integrins }\end{array}$ \\
\hline Porphyromonas gingivalis & $\beta 1$ integrins \\
\hline Shigella flexneri & $\beta 1$ integrins \\
\hline Staphylococcus aureus & $\begin{array}{l}\mathrm{FN}, \mathrm{LN}, \mathrm{Col} / \beta 1 \\
\text { integrins }\end{array}$ \\
\hline $\begin{array}{l}\text { Streptococcus pyogenes and } S . \\
\text { dysgalactiae }\end{array}$ & $\mathrm{FN} / \beta 1$ integrins \\
\hline $\begin{array}{l}\text { Yersinia pseudotuberculosis and } Y \text {. } \\
\text { enterocolitica }\end{array}$ & $\beta 1$ integrins \\
\hline \multicolumn{2}{|l|}{$\begin{array}{l}\text { Immunglobulin-related cell adhesion } \\
\text { molecules (IgCAMs) }\end{array}$} \\
\hline Haemophilus influenzae & CEACAMs \\
\hline Moraxella catarrhalis & CEACAMs \\
\hline $\begin{array}{l}\text { Neisseria gonorrhoeae and } N \text {. } \\
\text { meningitidis }\end{array}$ & CEACAMs \\
\hline \multicolumn{2}{|l|}{ Cadherins } \\
\hline Listeria monocytogenes & E-cadherin \\
\hline
\end{tabular}

CEACAMs, carcinoembryonic antigen-related cell adhesion molecules; Col, collagen; FN, fibronectin; LN, laminin; VN, vitronectin.

et al., 1999). Interference with fibronectin binding to the bacteria either by genetic deletion of FnBPs or by administration of a recombinant fibronectin-binding domain of FnBP-A as a competitive inhibitor during infections can attenuate staphylococcal virulence in several disease models such as abscess formation, mastitis, or endocarditis (Brouillette et al., 2003; Kuypers and Proctor, 1989; Menzies et al., 2002). However, the role of FnBPs in staphylococcal infections is not clear cut, and results from experimental models have been reported, where FnBPs did not contribute to the virulence of this pathogen (for review see Menzies, 2003). As staphylococci are associated with a large variety of clinical manifestations often involving secreted toxins, it is very likely that cell adhesion, and in particular FnBPs, will only act as bona fide virulence factors in some and not all of these situations. It is also conceivable that such FnBP-mediated adhesion and invasion processes are not only found in acute disease settings, but are of importance for the persistence of staphylococci in their host. Together, current experimental and epidemiologic data support the view that FnBPs contribute in some settings to the virulence of $S$. aureus and that cellular invasion via integrins represents 

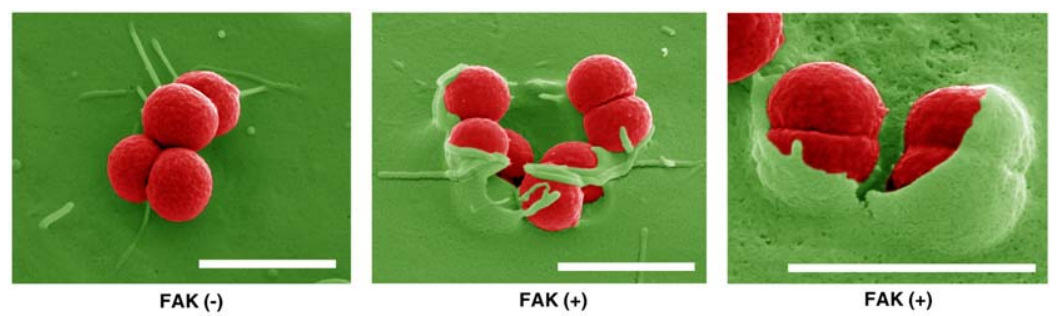

Fig. 1. FAK-deficient cells are resistant to cellular invasion by fibronectin-binding $S$. aureus via integrin $\alpha_{5} \beta_{1}$. FAK-deficient fibroblasts $(\mathrm{FAK}(-))$ or FAK $(-)$ cells re-expressing FAK $(\mathrm{FAK}(+))$ were infected for $1 \mathrm{~h}$ with $S$. aureus and analyzed by scanning electron microscopy. Pseudocolored (online version only) images depict bacteria in red and the fibroblast surface in green. Bars represent $2 \mu \mathrm{m}$ (figure is reproduced from Agerer et al. (2005) with permission).

one of the functional properties conferred by FnBP expression.

\section{Staphylococcal internalization via matrix binding integrins}

Interestingly, most integrins usually operate in the context of immobilized matrix proteins and are not considered to mediate endocytosis of attached ligands. In particular, integrin $\alpha_{5} \beta_{1}$, the fibronectin receptor, has been implied in organizing extracellular fibronectin into a fibrillar network by exerting force on the immobilized matrix protein (Schwarzbauer and Sechler, 1999). It is interesting to speculate that in contrast to immobilized fibronectin, the integrin-attached bacteria are pulled into the cell by the same cellular force-generating machinery that is used to remodel the fibronectin matrix under physiologic conditions. In general, extracellular matrix contact and integrin ligation induce the formation of protein complexes at the cytoplasmic part of cell adhesion sites. Prominent representatives of these integrin-mediated matrix adhesions include focal complexes, focal adhesions, and fibrillar adhesions that occur at discrete, focal spots along the matrix-facing surface of the adherent eukaryotic cell. These focal adhesion contacts have important structural and signaling functions as they dynamically link the clustered and ligand-bound integrins to the intracellular actin network that together with myosin is responsible for force generation (Zaidel-Bar et al., 2004). Due to their morphology and their functional connection to fibronectin fibril assembly, integrin $\alpha_{5} \beta_{1}$-initiated protein complexes have been termed fibrillar adhesions (Zamir and Geiger, 2001). A characteristic component of fibrillar adhesions is tensin, an actin-binding adaptor molecule (Zamir et al., 2000). Furthermore, fibrillar adhesions seem to accumulate less tyrosine-phosphorylated proteins when compared to other adhesive structures such as focal complexes, podosomes or mature focal adhesions (Zamir et al., 2000). Nevertheless, signaling enzymes, such as the protein tyrosine kinases Src and focal adhesion kinase (FAK) have been implied in the establishment of fibrillar adhesions and the integrin $\alpha_{5} \beta_{1}$-mediated assembly of a fibrillar fibronectin network (Ilic et al., 2004; Volberg et al., 2001). Recent work has started to address the role of these integrin-associated host cell factors for the FnBPmediated attachment and invasion of $S$. aureus. Engagement of integrin $\alpha_{5} \beta_{1}$ by fibronectin-binding staphylococci indeed induces the formation of fibrillar adhesion-like protein complexes at the site of bacterial attachment as characterized by the recruitment of tensin, FAK, zyxin, and vinculin (Agerer et al., 2005). Furthermore, interference with Src or FAK function abrogates the internalization of the bacteria via integrin $\alpha_{5} \beta_{1}$ and suppresses the increased tyrosine phosphorylation observed at bacterial attachment sites (Agerer et al., 2003, 2005; Fowler et al., 2003) (Fig. 1).

One of the effectors of activated FAK and Src kinases during integrin-mediated internalization has been identified as cortactin, an actin-binding protein that can associate with the Arp $2 / 3$ complex and promote actin polymerization, but that can also bind to dynamin-2, a regulator of membrane endocytosis (McNiven et al., 2000; Selbach and Backert, 2005). Together, these investigations support the view that fibronectin-coated staphylococci induce fibrillar adhesion-like contact sites which are regulated by protein tyrosine kinase signaling and link the bacteria-occupied integrins with the intracellular actin cytoskeleton. It is tempting to speculate that in this case bacterial internalization is promoted not only by increased actin polymerization generating membrane protrusions that enclose the pathogen, but at least partially by the contraction forces generated by the integrin-connected intracellular actinmyosin network that under physiological conditions promotes fibronectin fibrillogenesis.

\section{Integrin engagement during the infection process}

Though integrins are exploited by multiple bacterial and viral pathogens as well as parasites, it is not 
straightforward to conceive the role of this interaction in the context of an intact epithelium. In particular, integrins are distributed on the basolateral side of polarized epithelial cells that would be inaccessible for bacteria colonizing the throat, intestine or urogenital tract. Therefore, it has been speculated that these types of adhesins come into play only after the initial contact of the microbes with the tissue surface. As shown in several examples, pathogenic bacteria can influence the integrity of the epithelial barrier either directly by secreted toxins or indirectly by inducing granulocyte influx and tissue destruction (McCormick, 2003). Damage to the epithelial lining might then allow microbes to gain access to basolateral components such as integrins or cadherins.

\section{Exploitation of immunoglobulin-related cell adhesion molecules - the paradigm of pathogenic Neisseria species}

Both pathogenic neisserial species, $N$. gonorrhoeae and $N$. meningitidis, the gonococcus and the meningococcus, respectively, are highly specialized colonizers of the human mucosa. Gonococci and meningococci are known to express type IV pili, hair-like surface protrusions that provide both uncapsulated gonococci as well as capsulated meningococci with an evolutionarily optimized tool to establish the initial bacterial contact with the mucosal surfaces of the urogenital tract or the nasopharynx, respectively. Indeed, the thin and bendable pili are ideally suited for the task of long-range contact as they help to circumvent the electrostatic repulsion forces between the two negatively charged surfaces of the eukaryotic cell and the microbe. Moreover, type IV pili also seem to work in a manner similar to grappling hooks as the pilus fiber can be retracted by the bacterium (Maier et al., 2002). The result of pilus retraction by cell-associated bacteria, therefore, is a close apposition between the two membranes allowing additional, short-reach interactions to take place.

It is thought that at this point a second group of neisserial adhesins, the colony opacity-associated (Opa) proteins come into play (for review see Hauck and Meyer, 2003). Work over the last decade has identified the cellular receptors targeted by various Opa proteins. Historically, the first cellular receptor characterized was found to belong to the family of heparansulfate proteoglycans (HSPGs); however, only a limited set of Opa proteins with specificity for HSPGs $\left(\mathrm{Opa}_{\mathrm{HSPG}}\right.$ proteins) has been identified. Furthermore, Opa proteins with this type of specificity have not been described in meningococci. In contrast, the majority of the currently characterized meningococcal and gonococcal Opa proteins display binding specificity for human surface receptors of the carcinoembryonic antigen (CEA)related cell adhesion molecule (CEACAM) family (Opa $a_{\text {CEA }}$ proteins). All Opa ${ }_{\text {CEA }}$ proteins bind to the non-glycosylated $\mathrm{C}^{\prime} \mathrm{CFG}$-face of the immunoglobulindomain fold of the N-terminal domain of CEACAM1, CEACAM3, CEA (the product of the CEACAM5 gene), and CEACAM6 (formerly known as CD66a (CEACAM1), CD66c (CEACAM6), CD66d (CEACAM3), and CD66e (CEA)). Recently, the crystal structure of murine CEACAM1 has revealed a characteristic surface-exposed loop coordinated by Tyr-34 within the $\mathrm{C}^{\prime} \mathrm{CFG}$ face (Tan et al., 2002). It is assumed that a similar prominent surface extension helps to anchor Opa ${ }_{\text {CEA }}$ proteins to human CEACAM Nterminal domains, as mutagenesis of the corresponding Tyr-34 residue in human CEACAM1 abolished binding of $\mathrm{Opa}_{\mathrm{CEA}}$-expressing meningococci (Virji et al., 1999). It is interesting to point out that in mice CEACAM1 serves as the cellular receptor for mouse hepatitis virus (MHV) and MHV binding also takes place at the Nterminal domain (Dveksler et al., 1993). Importantly, the genetic ablation of the murine CEACAM1 Nterminal domain or of the complete molecule from the mouse genome has resulted in animals resistant to MHV infection pointing towards the essential role the pathogen-CEACAM interaction plays in this system (Blau et al., 2001; Hemmila et al., 2004).

CEACAMs are involved in cell-cell adhesion via both homotypic (CEACAM1, CEA, and CEACAM6) and/or heterotypic (CEA-CEACAM6 and CEACAM6-CEACAM8) interactions (Benchimol et al., 1989; Oikawa et al., 1991). Furthermore, CEACAM1 is implicated in hepatic uptake of insulin demonstrating that CEACAMs participate in internalization processes under physiological conditions (Poy et al., 2002a,b). An important aspect of CEACAM biology is the fact that several CEACAM family members can be expressed by epithelial cells, where they are usually located at the apical membrane of the polarized epithelium (Hammarstrom, 1999). This has important implications with respect to their role as bacterial receptors, as they are prominently exposed on mucosal surfaces and, therefore, accessible for incoming microbes. CEACAMs have also been linked to signal transduction into the cell and some isoforms seem to be connected to the cytoskeleton (Obrink, 1997; Schmitter et al., 2004). Binding of Opa ${ }_{\text {CEA }}$ proteins to CEACAM molecules is sufficient to induce the internalization of the bacteria into several cell types in vitro. On epithelial cells, CEACAM1, CEA, and CEACAM6 are often found to be co-expressed. In polarized T84 epithelial cell monolayers, CEA, CEACAM1, and CEACAM6 are transported apically, where they mediate invasion and subsequent transcytosis of Opa $\mathrm{CEA}_{\text {-express- }}$ ing gonococci (Wang et al., 1998).

It is important to point out that several other gramnegative, human-specific pathogens that share the same 
ecological niche and cause a similar spectrum of diseases as gonococci and meningococci have been found to possess CEACAM-binding adhesins. In an example of convergent evolution, typable and non-typable $H$. influenzae, $H$. influenzae biogroup aegyptius, and Moraxella catarrhalis have elaborated diverse surface antigens to engage CEACAMs (Hill and Virji, 2003; Virji et al., 2000). For example, $H$. influenzae contacts CEACAM family members by the outer membrane protein P5, whereas $M$. catarrhalis employs the UspA1 antigen (Hill et al., 2001; Hill and Virji, 2003). These findings imply that there must be some major advantage for bacteria colonizing the human mucosa to specifically target members of the CEACAM family. Novel insight suggests that in addition to providing a tight molecular anchor on the apical side of human epithelia, CEACAM recognition might serve an even more elaborate function in support of mucosal colonization. More specifically, CEACAM engagement by human pathogens appears to blunt an innate defense mechanism of stratified and squamous epithelial tissues, namely the exfoliation of superficial cells. Importantly, different non-opaque gonococcal variants cause detachment of epithelial cells after prolonged infection in culture, and such an exfoliation of urethral cells has been reported to occur during gonorrhea in vivo (Apicella et al., 1996; Evans, 1977; Melly et al., 1981; Mosleh et al., 1997; Ward et al., 1974). In striking contrast, prolonged infection with CEACAM-binding gonococci does not result in epithelial exfoliation (Muenzner et al., 2005). This process can be nicely documented by scanning electron microscopy, where upon infection of a confluent monolayer of a human cervix epithelial cell line with piliated, nonopaque or non-piliated $\mathrm{Opa}_{\mathrm{HSPG}}$-expressing gonococci, a loss in cell-cell contacts as well as the rounding and detachment of infected epithelial cells can be clearly observed (Fig. 2). However, when these cervix epithelial cells, that endogenously express CEACAM family members, are challenged with $\mathrm{Opa}_{\mathrm{CEA}}$-expressing gonococci, they show reduced cell-cell contacts, but still remain attached to the underlying extracellular matrix (Fig. 2). The lack of detachment was attributed to a
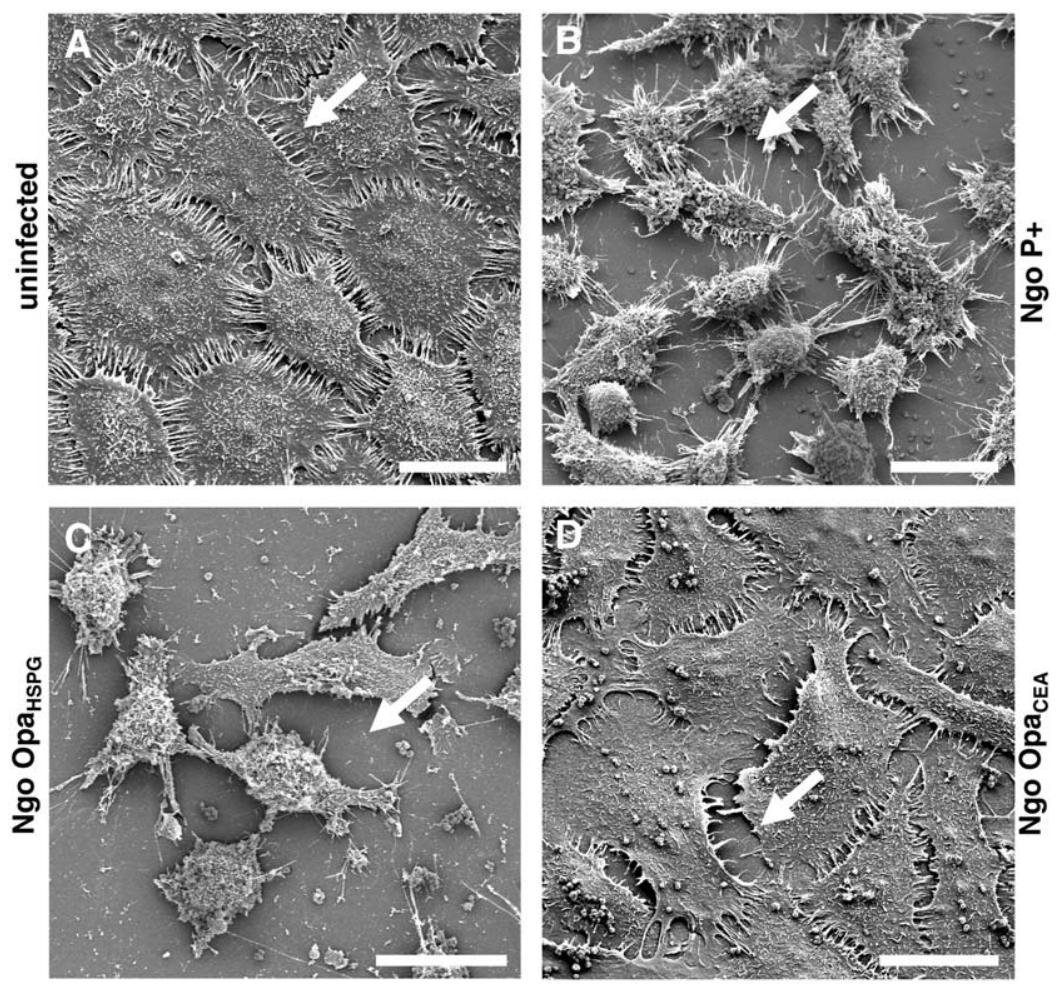

Fig. 2. Epithelial cells infected with CEACAM-binding Neisseria gonorrhoeae do not detach after prolonged infection. Confluent monolayers of a human cervix epithelial cell line (ME-180) grown on collagen-coated surfaces were (A) left uninfected, (B) infected

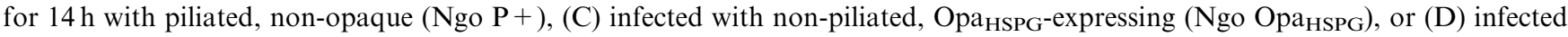

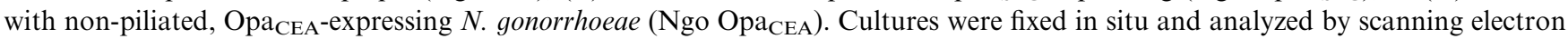
microscopy. Whereas uninfected monolayers display well spread epithelial cells with numerous cell-cell contacts (A, arrow), ME-180 cells infected with piliated or $\mathrm{Opa}_{\mathrm{HSPG}}$-expressing gonococci lose cell-cell contacts $(\mathrm{B}, \mathrm{C}$, arrow), round-up and detach from the extracellular matrix surface consistent with bacteria-induced exfoliation. In contrast, epithelial cells infected with CEACAMbinding gonococci $\left(\mathrm{Ngo}_{\mathrm{Opa}} \mathrm{PEA}_{\mathrm{C}}\right)$ stay attached to the matrix, though cell-cell contacts are diminished (D, arrow). Bars represent $20 \mu \mathrm{m}$ (figure is reproduced from Muenzner et al. (2005) with permission). 
dramatically increased adhesive property of cells infected with opaque Neisseria (Muenzner et al., 2005). By microarray-based gene expression analysis and further functional studies, the enhanced adhesive properties of the infected cells could be pinned down to the de novo expression of CD105 following CEACAM engagement. Importantly, upregulation of CD105 occurs in response to a number of CEACAM-binding human pathogens including $N$. gonorrhoeae, $N$. meningitidis, $H$. influenzae as well as $M$. catarrhalis and is sufficient to promote increased cell adhesion (Muenzner et al., 2005). Though further mechanistic studies are needed to elucidate how CD105 influences cell adhesion, first results suggest that the presence of CD105 has a positive impact on the activation status of matrix-binding integrins (Muenzner et al., 2005). These results imply that integrins not only serve as a gateway into eukaryotic cells, as discussed above in the case of $S$. aureus, but they can be also indirectly exploited by bacteria to modulate the adhesive properties of the infected host cell. Together, it is conceivable that pathogen-initiated CEACAM stimulation, the increased expression of CD105, and the ensuing enhanced cell-matrix adhesion are central events that counteract the exfoliation of infected epithelial cells in vivo and, therefore, facilitate the colonization of the human mucosa by CEACAM-binding bacteria. In light of these results, it is tempting to speculate that the prevention of epithelial detachment attained through CEACAM binding is the major evolutionary driving force behind the appearance of distinct CEACAMdirected adhesins in several bacterial species colonizing the human mucosa.

\section{Conclusions}

Pathogen-eukaryotic cell recognition and the tight attachment of microorganisms to their host are of fundamental importance in major infectious diseases. Work over the last decade has identified numerous adhesin-receptor pairs, and these molecular investigations still yield exciting and often surprising insights. In many cases, pathogenic microorganisms target cell adhesion molecules to intimately interact with target cells and to trigger specific responses upon receptor engagement. The more we learn about the intricate molecular communication taking place at the bacterial-host cell interface, the more it is becoming evident that cell adhesion molecule recognition by bacterial adhesins has effects beyond the attachment to the cell surface or internalization into the host cell. The coming years will witness an increased appreciation and understanding of such postadhesion events that modulate and shape the infection process.

\section{Acknowledgement}

The authors would like to thank the BMBF (01 KI 8906/0) and the Deutsche Forschungsgemeinschaft (Ha2568/3-2) for financial support and the members of the lab for suggestions on this manuscript.

\section{References}

Agerer, F., Michel, A., Ohlsen, K., Hauck, C.R., 2003. Integrin-mediated invasion of Staphylococcus aureus into human cells requires Src family protein tyrosine kinases. J. Biol. Chem. 278, 42524-42531.

Agerer, F., Lux, S., Michel, A., Rohde, M., Ohlsen, K., Hauck, C.R., 2005. Cellular invasion by Staphylococcus aureus reveals a functional link between focal adhesion kinase and cortactin in integrin-mediated internalisation. $\mathbf{J}$. Cell Sci. 118, 2189-2200.

Apicella, M.A., Ketterer, M., Lee, F.K.N., Zhou, D., Rice, P.A., Blake, M.S., 1996. The pathogenesis of gonococcal urethritis in men: confocal and immunoelectron microscopic analysis of urethral exsudates from men infected with Neisseria gonorrhoeae. J. Infect. Dis. 173, 636-646.

Benchimol, S., Fuks, A., Jothy, S., Beauchemin, N., Shirota, K., Stanners, C.P., 1989. Carcinoembryonic antigen, a human tumor marker, functions as an intercellular adhesion molecule. Cell 57, 327-334.

Blau, D.M., Turbide, C., Tremblay, M., Olson, M., Letourneau, S., Michaliszyn, E., Jothy, S., Holmes, K.V., Beauchemin, N., 2001. Targeted disruption of the Ceacam1 (MHVR) gene leads to reduced susceptibility of mice to mouse hepatitis virus infection. J. Virol. 75, 8173-8186.

Brouillette, E., Grondin, G., Shkreta, L., Lacasse, P., Talbot, B.G., 2003. In vivo and in vitro demonstration that Staphylococcus aureus is an intracellular pathogen in the presence or absence of fibronectin-binding proteins. Microb. Pathog. 35, 159-168.

Dveksler, G.S., Dieffenbach, C.W., Cardellichio, C.B., McCuaig, K., Pensiero, M.N., Jiang, G.S., Beauchemin, N., Holmes, K.V., 1993. Several members of the mouse carcinoembryonic antigen-related glycoprotein family are functional receptors for the coronavirus mouse hepatitis virus-A59. J. Virol. 67, 1-8.

Evans, B.A., 1977. Ultrastructural study of cervical gonorrhea. J. Infect. Dis. 136, 248-255.

Foster, T.J., Hook, M., 1998. Surface protein adhesins of Staphylococcus aureus. Trends Microbiol. 6, 484-488.

Fowler, T., Johansson, S., Wary, K.K., Hook, M., 2003. Src kinase has a central role in in vitro cellular internalization of Staphylococcus aureus. Cell. Microbiol. 5, 417-426.

Hammarstrom, S., 1999. The carcinoembryonic antigen (CEA) family: structures, suggested functions and expression in normal and malignant tissues. Semin. Cancer Biol. 9, 67-81.

Hauck, C.R., Meyer, T.F., 2003. Small talk: Opa proteins as mediators of Neisseria-host-cell communication. Curr. Opin. Microbiol. 6, 43-49. 
Hemmila, E., Turbide, C., Olson, M., Jothy, S., Holmes, K.V., Beauchemin, N., 2004. Ceacam1a-/- mice are completely resistant to infection by murine coronavirus mouse hepatitis virus A59. J. Virol. 78, 10156-10165.

Hill, D.J., Virji, M., 2003. A novel cell-binding mechanism of Moraxella catarrhalis ubiquitous surface protein UspA: specific targeting of the $\mathrm{N}$-domain of carcinoembryonic antigen-related cell adhesion molecules by UspA1. Mol. Microbiol. 48, 117-129.

Hill, D.J., Toleman, M.A., Evans, D.J., Villullas, S., Van Alphen, L., Virji, M., 2001. The variable P5 proteins of typeable and non-typeable Haemophilus influenzae target human CEACAM1. Mol. Microbiol. 39, 850-862.

Ilic, D., Kovacic, B., Johkura, K., Schlaepfer, D.D., Tomasevic, N., Han, Q., Kim, J.B., Howerton, K., Baumbusch, C., Ogiwara, N., Streblow, D.N., Nelson, J.A., Dazin, P., Shino, Y., Sasaki, K., Damsky, C.H., 2004. FAK promotes organization of fibronectin matrix and fibrillar adhesions. $\mathbf{J}$. Cell Sci. 117, 177-187.

Kuypers, J.M., Proctor, R.A., 1989. Reduced adherence to traumatized rat heart valves by a low-fibronectin-binding mutant of Staphylococcus aureus. Infect. Immun. 57, 2306-2312.

Maier, B., Potter, L., So, M., Long, C.D., Seifert, H.S., Sheetz, M.P., 2002. Single pilus motor forces exceed 100 pN. Proc. Natl. Acad. Sci. USA 99, 16012-16017.

McCormick, B.A., 2003. The use of transepithelial models to examine host-pathogen interactions. Curr. Opin. Microbiol. 6, 77-81.

McNiven, M.A., Kim, L., Krueger, E.W., Orth, J.D., Cao, H., Wong, T.W., 2000. Regulated interactions between dynamin and the actin-binding protein cortactin modulate cell shape. J. Cell Biol. 151, 187-198.

Melly, M.A., Gregg, C.R., McGee, Z.A., 1981. Studies of toxicity of Neisseria gonorrhoeae for human fallopian tube mucosa. J. Infect. Dis. 143, 423-431.

Menzies, B.E., 2003. The role of fibronectin binding proteins in the pathogenesis of Staphylococcus aureus infections. Curr. Opin. Infect. Dis. 16, 225-229.

Menzies, B.E., Kourteva, Y., Kaiser, A.B., Kernodle, D.S., 2002. Inhibition of staphylococcal wound infection and potentiation of antibiotic prophylaxis by a recombinant fragment of the fibronectin-binding protein of Staphylococcus aureus. J. Infect. Dis. 185, 937-943.

Mosleh, I.M., Boxberger, H.J., Sessler, M.J., Meyer, T.F., 1997. Experimental infection of native human ureteral tissue with Neisseria gonorrhoeae: adhesion, invasion, intracellular fate, exocytosis, and passage through a stratified epithelium. Infect. Immun. 65, 3391-3398.

Muenzner, P., Rohde, M., Kneitz, S., Hauck, C.R., 2005. CEACAM engagement by human pathogens enhances cell adhesion and counteracts bacteria-induced detachment of epithelial cells. J. Cell Biol. 170, 825-836.

Obrink, B., 1997. CEA adhesion molecules: multifunctional proteins with signal-regulatory properties. Curr. Opin. Cell Biol. 9, 616-626.

Oikawa, S., Inuzuka, C., Kuroki, M., Arakawa, F., Matsuoka, Y., Kosaki, G., Nakazato, H., 1991. A specific heterotypic cell adhesion activity between members of carcinoembry- onic antigen family, W272 and NCA, is mediated by $\mathrm{N}$ domains. J. Biol. Chem. 266, 7995-8001.

Ozeri, V., Rosenshine, I., Mosher, D.F., Fassler, R., Hanski, E., 1998. Roles of integrins and fibronectin in the entry of Streptococcus pyogenes into cells via protein F1. Mol. Microbiol. 30, 625-637.

Patti, J.M., Allen, B.L., McGavin, M.J., Hook, M., 1994. MSCRAMM-mediated adherence of microorganisms to host tissues. Annu. Rev. Microbiol. 48, 585-617.

Poy, M.N., Ruch, R.J., Fernstrom, M.A., Okabayashi, Y., Najjar, S.M., 2002a. Shc and CEACAM1 interact to regulate the mitogenic action of insulin. J. Biol. Chem. 277, 1076-1084.

Poy, M.N., Yang, Y., Rezaei, K., Fernstrom, M.A., Lee, A.D., Kido, Y., Erickson, S.K., Najjar, S.M., 2002b. CEACAM1 regulates insulin clearance in liver. Nat. Genet. 30, 270-276.

Schmitter, T., Agerer, F., Peterson, L., Muenzner, P., Hauck, C.R., 2004. Granulocyte CEACAM3 is a phagocytic receptor of the innate immune system that mediates recognition and elimination of human-specific pathogens. J. Exp. Med. 199, 35-46.

Schwarzbauer, J.E., Sechler, J.L., 1999. Fibronectin fibrillogenesis: a paradigm for extracellular matrix assembly. Curr. Opin. Cell Biol. 11, 622-627.

Schwarz-Linek, U., Werner, J.M., Pickford, A.R., Gurusiddappa, S., Kim, J.H., Pilka, E.S., Briggs, J.A., Gough, T.S., Hook, M., Campbell, I.D., Potts, J.R., 2003. Pathogenic bacteria attach to human fibronectin through a tandem beta-zipper. Nature 423, 177-181.

Selbach, M., Backert, S., 2005. Cortactin: an Achilles' heel of the actin cytoskeleton targeted by pathogens. Trends Microbiol. 13, 181-189.

Sinha, B., Francois, P.P., Nusse, O., Foti, M., Hartford, O.M., Vaudaux, P., Foster, T.J., Lew, D.P., Herrmann, M., Krause, K.H., 1999. Fibronectin-binding protein acts as Staphylococcus aureus invasin via fibronectin bridging to integrin alpha5beta1. Cell. Microbiol. 1, 101-117.

Tan, K., Zelus, B.D., Meijers, R., Liu, J.H., Bergelson, J.M., Duke, N., Zhang, R., Joachimiak, A., Holmes, K.V., Wang, J.H., 2002. Crystal structure of murine sCEACAM1a[1,4]: a coronavirus receptor in the CEA family. EMBO J. 21, 2076-2086.

Virji, M., Evans, D., Hadfield, A., Grunert, F., Teixeira, A.M., Watt, S.M., 1999. Critical determinants of host receptor targeting by Neisseria meningitidis and Neisseria gonorrhoeae: identification of Opa adhesiotopes on the $\mathrm{N}$-domain of CD66 molecules. Mol. Microbiol. 34, 538-551.

Virji, M., Evans, D., Griffith, J., Hill, D., Serino, L., Hadfield, A., Watt, S.M., 2000. Carcinoembryonic antigens are targeted by diverse strains of typable and non-typable Haemophilus influenzae. Mol. Microbiol. 36, 784-795.

Volberg, T., Romer, L., Zamir, E., Geiger, B., 2001. pp60(csrc) and related tyrosine kinases: a role in the assembly and reorganization of matrix adhesions. J. Cell Sci. 114, 2279-2289.

Wang, J., Gray-Owen, S.D., Knorre, A., Meyer, T.F., Dehio, C., 1998. Opa binding to cellular CD66 receptors mediates the transcellular traversal of Neisseria gonorrhoeae across polarized T84 epithelial cell monolayers. Mol. Microbiol. $30,657-671$. 
Ward, M.E., Watt, P.J., Robertson, J.N., 1974. The human fallopian tube: a laboratory model for gonococcal infection. J. Infect. Dis. 129, 650-659.

Zaidel-Bar, R., Cohen, M., Addadi, L., Geiger, B., 2004. Hierarchical assembly of cell-matrix adhesion complexes. Biochem. Soc. Trans. 32, 416-420.
Zamir, E., Geiger, B., 2001. Molecular complexity and dynamics of cell-matrix adhesions. J. Cell Sci. 114, 3583-3590.

Zamir, E., Katz, M., Posen, Y., Erez, N., Yamada, K.M., Katz, B.Z., Lin, S., Lin, D.C., Bershadsky, A., Kam, Z., Geiger, B., 2000. Dynamics and segregation of cell-matrix adhesions in cultured fibroblasts. Nat. Cell Biol. 2, 191-196. 